\title{
Persistent Active Longitudes in Sunspot Activity: Sun-as-a-Star Approach
}

\author{
S. V. Berdyugina \\ Institute of Astronomy, ETHZ, 8092 Zurich, Switzerland \\ Astronomy Division, PO Box 3000, 90014 University of Oulu, Finland \\ I. G. Usoskin \\ Sodankylä Geophysical Observatory (Oulu unit), 90014 University of
Oulu, Finland
}

\begin{abstract}
Using a new Sun-as-a-star approach we analyze sunspot group data for the past 120 years and reveal that sunspots are formed preferably in two persistent migrating active longitudes $180^{\circ}$ apart. Their migration is determined by changes of the mean latitude of sunspots and the surface differential rotation. The two active regions periodically alternate being the dominant region with a period of about 3.7 years similar to the "flip-flop" phenomenon known in starspot activity. The fact that the Sun shows the same pattern of magnetic activity as highly active stars strengthens the solar paradigm for magnetic activity on cool stars.
\end{abstract}

\section{Introduction}

The distribution of sunspots in the solar photosphere reflects the distribution of magnetic fields in the convection zone and provides strong observational constraints on the solar dynamo theory. Sunspots are known to appear preferably in narrow latitudinal belts and approach the equator as the solar 11-year cycle advances. Untill recently preferred longitudes of sunspot formation, so-called active longitudes, were found only on time-scales of 1-3 years (e.g., Vitinskij 1969) or 1-2 solar cycles (Balthasar \& Schüssler 1983). On the other hand, persistent active longitudes have been detected on different types of active stars, including a young solar analogue (Berdyugina \& Tuominen 1998; Korhonen, Berdyugina \& Tuominen 2002; Berdyugina, Pelt, \& Tuominen 2002). On single stars they have been found to migrate in phase with a varying rate, seemingly due to surface differential rotation. Since a similar behaviour can be expected for the Sun, we have abandoned the assumption on a constant rotation rate of solar active longitudes, made by previous investigators, and for the first time revealed active longitudes in sunspot activity on the century time scale (Berdyugina \& Usoskin 2003).

Here we analyze sunspot group data for the past 120 years with a new sunas-a-star approach which allows us to study the Sun as we would study a distant star whose large-scale magnetic activity only can be resolved. Our technique is 

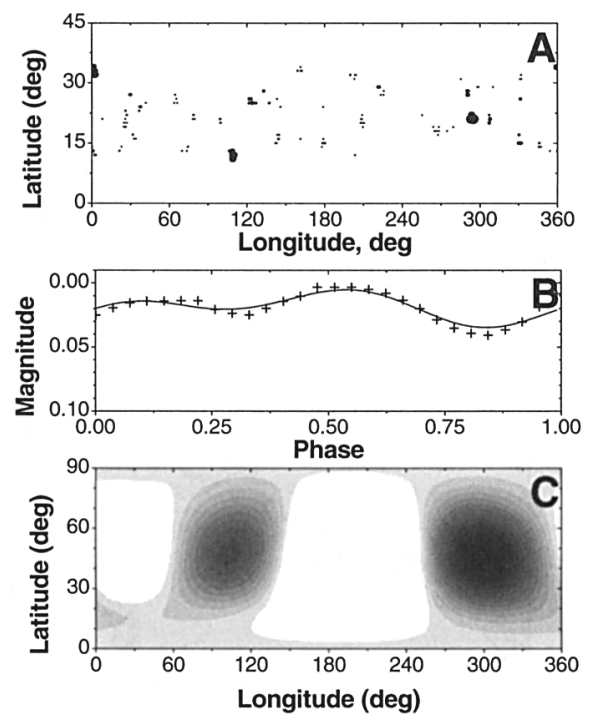

Figure 1. An illustration of the sun-as-a-star approach based on the synthesis and analysis of solar light curves. Sunspots observed in the northern hemisphere during the Carrington rotation 1808 (October to November 1988) are shown in panel $\mathbf{A}$, the synthesized (crosses) and restored (solid line) solar light curves in panel $\mathbf{B}$, and the restored image of the spot filling factor in panel $\mathbf{C}$.

sensitive to active longitudes and provides the possibility for a direct comparison of the Sun with active cool stars.

\section{Active longitudes and differential rotation}

We use daily data on sunspot group locations and areas collected at the Royal Greenwich Observatory, the US Air Force and National Oceanic and Atmospheric Administration for the years 1874-2001, covering 11 full solar cycles. Our new sun-as-a-star approach is based on the inversion method having been developed for an analysis of light curves of active cool stars (Berdyugina et al. $2002)$. For each Carrington solar rotation $(\approx 27$ days) we build a temperature distribution defined by cool dark spots of $4000 \mathrm{~K}$ on the bright surface of $5750 \mathrm{~K}$ (Fig. 1A). We assume that during one Carrington rotation period the solar surface rotates rigidly and sunspots are stable. Then, from the temperature matrix, we synthesize a bolometric light curve, smoothing the longitudinal variation in temperature (Fig. 1B). Because of the known asymmetry between the northern and southern hemispheres, we treat them separately. Then, applying the Occamian inversion method (Berdyugina 1998) to the synthesized light curve, we reconstruct images of sunspot distribution by means of the probability of a spot to exist at each location, so-called spot filling factor (Fig. 1C). Since a light curve is one-dimensional, the reconstructed image contains only the longitudinal information (Berdyugina et al. 2002). Maxima of the spot filling factor give the 

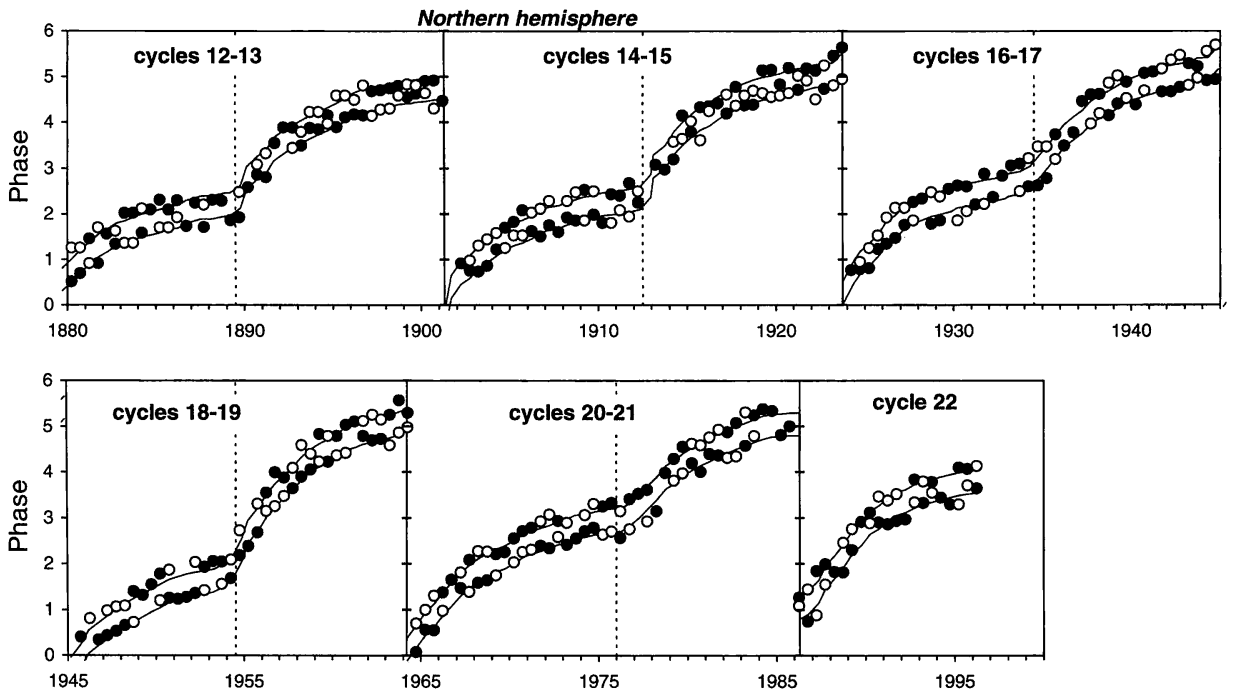

Figure 2. Semi-annual phases of regions of preferred sunspot formation for solar cycles 12 to 22 in the northern hemisphere. Filled circles denote the phases of the active longitude, which is dominant during the corresponding 6 months, and open circles denote the other active longitude. Curves depict the phase migration resulting from a combination of changes of the mean latitude of sunspots and the differential rotation. Vertical lines separate the solar cycles. The migration continues over all 11 cycle and the active longitude delay for about 2.5 rotations per cycle. For better visibility the migration in the plots is discontinued every second cycle. Similar structures are found in the southern hemisphere as well.

most probable longitudes of spot formation regions and correspond to the minimum brightness and largest spot concentrations. Therefore, from each image we derive Carrington longitudes of local maxima of the spot filling factor, which we transformed into fractions (phases) of the Carrington period. We find two long-lived activity regions (active longitudes) separated by about $180^{\circ}$, which migrate in phase and lag for 2-3 rotations over a solar cycle. The two active longitudes are more apparent if the phases are semi-annually averaged with weights proportional to the corresponding maximum bolometric magnitudes (Fig. 2).

In order to estimate the significance of the two active longitudes, we calculate histograms of locations of recovered activity regions with respect to the semi-annual averages of only one active longitude (Fig. 3). The two-peak distribution of the locations confirms the presence of the two active longitudes separated by half a period. The nearly Gaussian shape of the peaks implies that individual spots are randomly spread around the preferred longitudes.

The rate of migration of active longitudes varies over the solar cycle (Fig. 2 ), suggesting that the migration follows the surface differential rotation as the mean latitude of sunspots changes during the cycle. The surface angular velocity at a given latitude $\psi$ is described by $\Omega=A+B \sin ^{2} \psi$, where $A$ is the rotation 


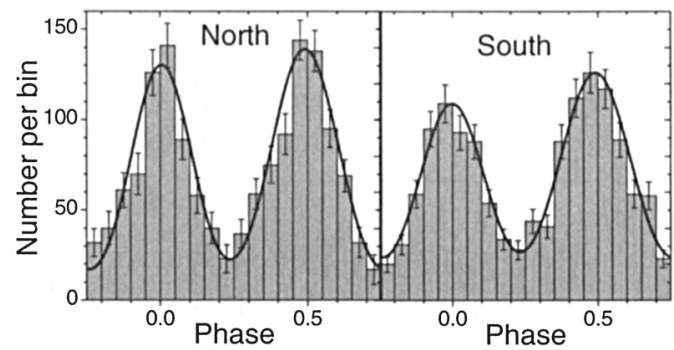

Figure 3. Histogram distribution of recovered spot locations with respect to the semi-annual averages of one active longitude for the solar cycles 12 to 22 . Error bars correspond to the $1 \sigma$ statistical error. The distribution is described by a double Gaussian. For both hemispheres, the means of the two Gaussians are 0.0 and 0.5 , and the standard deviation is about 0.14 .

rate at the equator and $B$ is the differential rotation rate. The latter can be determined from the observed phase lags of the active longitudes. An expected lag in Carrington longitude, $\Delta \lambda(\Delta t)$, is determined by changes of the mean latitude $\psi$ during the time interval $\Delta t$ as follows: $\Delta \lambda(\Delta t)=B \int_{\Delta t} \sin ^{2} \psi(t) d t$, with $B$ being the differential rotation rate in question. Using semi-annual mean latitudes of sunspots obtained from the original data, we have calculated values of the integral starting from the beginning of each cycle. Comparing them with the observed lags in longitude, $\Delta \lambda$, for all cycles, we find $B=-3.45 \pm 0.04 \mathrm{deg} / \mathrm{day}$, in agreement with the value of $-3.43 \mathrm{deg} / \mathrm{day}$ obtained from SOHO/MDI measurements (Schou et al. 1998). This confirms that the migration is determined by changes of the mean latitude of sunspots and the differential rotation, implying that both longitudinal and latitudinal migrations are interrelated.

\section{Flip-flop effect}

We noticed that spots are preferably concentrated in one active longitude, which is dominant at a given time (Fig. 2). The dominant activity switches periodically between the active longitudes. This alternation is similar to the "flip-flop" phenomenon known in stellar activity (Berdyugina \& Tuominen 1998). In order to describe this phenomenon quantitatively, we build a series of the activity dominance, $D$, so that for each Carrington rotation either $D=0$, if the longitude at earlier phase dominates, or $D=1$, if the other longitudes dominates. By calculating a yearly running mean, we smoothed the discrete $D$-series into a continuous series which suggests the presence of a periodic pattern in the dominance (Fig. 4a). The power spectrum of the smoothed $D$-series (Fig. $4 \mathrm{~b}$ ) reveals peaks at periods of about 3.65 and 3.8 years in the southern and northern hemispheres, respectively. This implies that the two longitudes alternate periodically the dominant activity in about 1.8 years. Two consecutive switches compose the 3.7-year period, which is nearly $1 / 3$ of the 11 -year sunspot cycle. The same relation between analogous cycles have been found for a very active young star (Berdyugina et al. 2002), that suggests similar dynamo processes in the Sun and other active stars. 

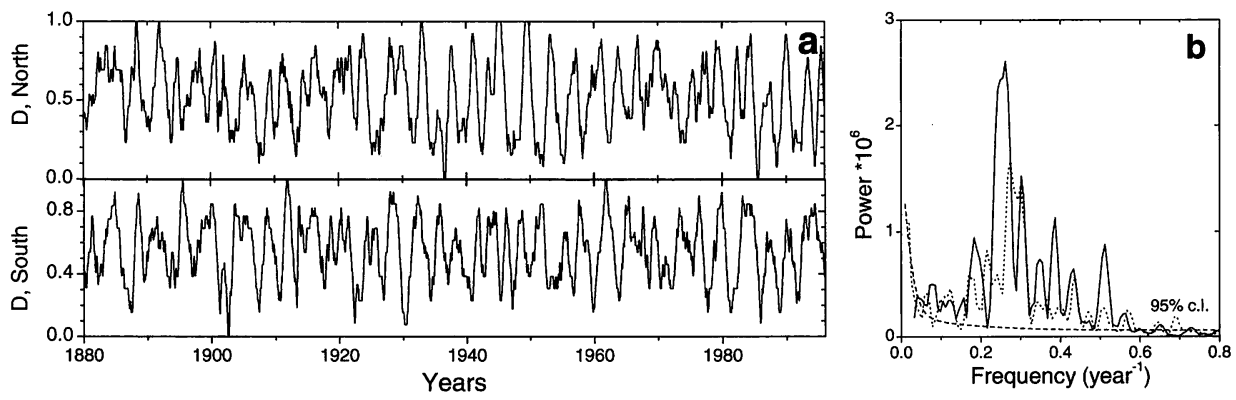

Figure 4. Yearly smoothed dominance of the two active longitudes in the northern and southern hemispheres (a) and corresponding power spectra (b). The dashed line in the panel $\mathbf{b}$ indicates the $95 \%$ confidence level.

\section{Conclusions}

Sunspots in both northern and southern hemispheres are preferably formed around two active longitudes separated by $180^{\circ}$. For the first time, we showed that they are persistent for at least 120 years.

The two active longitudes is a long-lived quasi-rigid structure, which continuously migrate with respect to a chosen reference frame with a variable rate. The migration of the active longitudes is caused by changes of the mean latitude of the sunspot formation and the differential rotation. The rate of the differential rotation $-3.46 \mathrm{degr} /$ day obtained from the migration rate is in agreement with the recent measurements by SOHO/MDI.

The major spot activity alternates the active longitudes in about 1.8 years. In the northern and southern hemispheres this results in the flip-flop cycles of 3.8 and 3.65 years, respectively, which is about $1 / 3$ of the sunspot 11 -yr cycle.

The fact that the Sun shows the same pattern of magnetic activity as highly active stars strengthens the solar paradigm for magnetic activity on cool stars, allowing the physics of magnetic activity on such stars to be understood within the context of the physical principles deduced from the study of the Sun.

\section{References}

Balthasar, H. \& Schüssler, M. 1983, Sol. Phys., 87, 23

Berdyugina, S. V. 1998, A\&A, 338, 97

Berdyugina, S. V. \& Tuominen, I. 1998, A\&A, 336, L25

Berdyugina, S. V. \& Usoskin, I. 2003, A\&A, 405, 1121

Berdyugina, S.V., Pelt, J., \& Tuominen, I. 2002, A\&A, 394, 505

Korhonen, H., Berdyugina, S. V., \& Tuominen, I. 2002, A\&A, 390, 179

Schou, J. et al. 1998 ApJ, 505, 390

Vitinskij, Yu. I. 1969, Sol. Phys., 7, 210 